\title{
Emitters and Receivers for Impulse Radio Ultra- WideBand and Their Healthcare Applications
}

\author{
R. Vauche ${ }^{1}$, S. Bourdel ${ }^{2}$, J. Gaubert ${ }^{1}$, N. Dehaese ${ }^{1}$, H. Barthelemy ${ }^{1}$ \\ ${ }^{1}$ Aix Marseille Université, CNRS, Université de Toulon, IM2NP UMR 7334 Marseille, France \\ ${ }^{2}$ Univ. Grenoble Alpes, IMEP-LAHC, F-38000 Grenoble, France
}

\begin{abstract}
Healthcare applications requirements are presented in this paper and a state-of-the-art analysis shows that Impulse Radio Ultra-WideBand (IR-UWB) is particularly suitable in terms of energy efficiency for this type of short range communications for which different data rates are needed. Power saving strategies for emitters and receivers are then discussed and three examples of implementations are presented with their measurement results. The first is a versatile transmitter with tunable bandwidth which exhibits 0.9Vpp pulses and a power consumption of at least $11.03 \mathrm{~mW}$ at $50 \mathrm{Mbps}$. The second is a 3.110.6GHz transmitter with a power management scheme implemented on-chip which exhibits $2 \mathrm{Vpp}$ pulses and a power consumption of $7.3 \mu \mathrm{W}$ at $100 \mathrm{kbps}$ and $3.4 \mathrm{~mW}$ at $100 \mathrm{Mbps}$. The last is a non-coherent $3-5 \mathrm{GHz}$ receiver with $-89 \mathrm{dBm}$ sensitivity at $100 \mathrm{kbps}$ and $144 \mathrm{pJ} / \mathrm{bit}$ energy consumption when used in a power gated 100Mbps burst mode.
\end{abstract}

Keywords-Healthcare Applications; Impulse Radio; Power Gating; Data Rate Management; UWB; Transceiver

\section{INTRODUCTION}

Modern biomedical monitoring or stimulation systems use wireless communications to avoid the use of wires which is uncomfortable for the patient and the medical workers, and reduces the reliability of the overall system. The needs in biomedical application are very specific and depend on the monitored parameters or the stimulated parts [1][2][3]. As presented in TABLE I. , physiological monitoring needs low or medium data rates and can be realized with an unidirectional link, whereas next generation of brain machine interfaces needs a large number of interconnections which leads to high data rates up to $100 \mathrm{Mbps}$ [3]. Concerning the power consumption, the needs are also very widespread since the transmitter life can vary from few hours for ElectroCardioGraphy (ECG) to few years when in-body stimulation systems are considered. The communication range appears to be the most relaxed constraint since the on-body or in-body transmitters are close to the receiver which can consist of a simple hub that concentrates the data and sends it to a remote monitoring system.

Due to this large variety of needs, several wireless technologies have been used to implement biomedical monitoring and stimulation systems as: i) High Frequency Radio Frequency IDentification (HF-RFID; 13.56MHz) [4][5], ii) Ultra-High Frequency RFID (UHF-RFID; 900MHz) [6][7], iii) $400 \mathrm{MHz}$ Narrow Band (400MHz-NB) [8][9][10], iv) 2.45GHz-NB [11][12][13], and v) Impulse Radio Ultra-
WideBand (IR-UWB) [14][15][16]. The most encountered systems are based on HF-RFID and 400MHz-NB technologies which are the most mature. Moreover, biomedical standards have been specified for the 400MHz-NB whereas HF-RFID is quite attractive since remotely powered transponders can be achieved with commercial off the shelf RFID devices. However, 2.45GHz-NB systems are recently emerging essentially due to the growth of Bluetooth Low Energy (BLE) [12][13] and the ease of interconnecting the transponder with standardized systems.

In the field of biomedical applications, IR-UWB appears first to be a new comer since it seems to offer a large number of advantages such as low power consumptions, high data rates or low radiated power which allows IR-UWB to comply with Specific Absorption Rate (SAR) specifications [14]. As shown in Fig. 1 which compares four essential performances of a wireless link (ie. range, data rate, TX and RX energy efficiency in bit/nJ equals to the data rate divided by TX and RX power consumption), NB technologies are the best suited to achieve long range and data rates around $1 \mathrm{Mbps}$. RFID systems appears to be the best suited when ultra-low power is needed since it can operate without battery but it fails to achieve medium or high data rates and are quite limited in terms of range (lower than $1 \mathrm{~m}$ for HF-RFID systems). IRUWB is a technology that allows both high and low data rates communications at low power. This is due to the ease of duty cycling transceivers which allows the energy efficiency to be always maximized. However, IR-UWB can only achieve short range communications when non-coherent receivers are used but this is not critical for the considered applications.

This paper is organized as follows. Section II deals with power saving strategies for IR-UWB emitters and receivers, and provides design considerations and power management schemes. In section III, prototypes of two emitters and of one receiver are presented with their measurement results and their expected power saving abilities.

\section{Power SAVING StRATEGIES IN IR-UWB}

IR-UWB systems use pulse modulations to transmit data and the emitted symbols can also be coded by the presence or the absence of a particular pulse at a particular time. Since pulses duration (few ns) in UWB is generally short compared to the mean Pulse Repetition Period (PRP MEAN ), the power consumed by an IR-UWB emitter (resp. receiver) can be considered equal to a constant power $\mathrm{P}_{\mathrm{DC}}$ when no pulse is emitted (resp. received). Otherwise, an extra energy $E_{A C}$ is 
consumed when a pulse has to be emitted (resp. received). In IR-UWB, it is also possible to write the power consumption $\mathrm{P}_{\mathrm{C}}$ as follows:

$$
P_{C}=P_{D C}+E_{A C} \times P R F_{M E A N}
$$

where $\mathrm{PRF}_{\mathrm{MEAN}}$ is the mean Pulse Repetition Frequency (PRF).

\section{A. Pulse Modulation Choice}

The dynamic part $\mathrm{P}_{\mathrm{AC}}$ of the power consumption $\mathrm{P}_{\mathrm{C}}$ can be written as a function of the bit rate $\mathrm{D}_{\mathrm{b}}$ (bit/s) as follows:

$$
P_{A C}=E_{A C} \times P R F_{M E A N}=E_{A C} \times D_{b} / N
$$

where $\mathrm{N}$ is the mean number of bits convoyed by pulse which depends on the modulation used. To reduce the $\mathrm{P}_{\mathrm{AC}}$, a tradeoff must be done. On the TX side, the higher $\mathrm{N}$ is and the lower the $\mathrm{P}_{\mathrm{AC}}$ is. On the $\mathrm{RX}$ side, a high $\mathrm{N}$ increases the complexity and reduces the $\mathrm{P}_{\mathrm{AC}}$. Thus, On-Off Keying (OOK) type modulations $(\mathrm{N}=2)$ is a good compromise and can be demodulated with simple non-coherent receivers whose power consumption is less than the coherent receivers [17]. Moreover, standard OOK can be replaced by the Randomly Alternate OOK (RA-OOK) defined in [14] which allows the authorized bit rate and/or range to be increased compared to BPSK at the same mean Equivalent Isotropically Radiated Power (EIRP) thanks to the properties of pulse modulations [14].

\section{B. Design Considerations for IR-UWB emitters}

In IR-UWB, emitters are based on pulse generators which emit a pulse according to their control inputs when an active edge is provided on their trigger input. However, the pulse generator inputs need to be controlled with the help of a baseband manager in order to implement pulse modulations. For usual Pulse Amplitude Modulations (PAM) as OOK, the baseband manager requires only CLOCK and DATA digital inputs to provide the input signals needed by the pulse generator and its required trigger signal.

By using the following definition of the emitted power $\mathrm{P}_{\mathrm{E}}$ :

$$
P_{E}=E_{P} \times P R F_{M E A N}
$$

where $E_{P}$ is the emitted energy by each pulse, it is possible to defined the power efficiency $\eta_{P}$ of an IR-UWB emitter as follows:

$$
\eta_{P}=\frac{P_{E}}{P_{C}}=\frac{E_{P} \times P R F_{M E A N}}{P_{D C}+E_{A C} \times P R F_{M E A N}}
$$

When this equation is differentiated with respect to

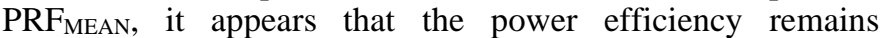
constant and maximized regardless of PRF $F_{\text {MEAN }}$ if $P_{D C}$ is null. Thus, to optimize the power efficiency of an IR-UWB emitter, it is necessary to minimize $\mathrm{P}_{\mathrm{DC}}$ by using functions for which most of the power consumption is dynamic and depends on the number of switching per second as CMOS logic gates, or by using fast on/off switchable architectures [18] which can be easily implemented since pulse generation times are known from the pulse generator point of view on contrary to the receiver side. However, it exists a particular value of $P_{R F_{M E A N}}$ for which $\mathrm{P}_{\mathrm{DC}}$ can be considered negligible compared to $\mathrm{P}_{\mathrm{AC}}$ without the use of power saving methods. Depending on the bit rate needs, it is critical to reduce the $\mathrm{P}_{\mathrm{DC}}$ for energy efficiency optimization. Thus, for low rate applications, this parameter is the most limiting as far as the power consumption is concerned.

To illustrate this subsection, the design of an all-digital pulse generator, where different logic families can be used as CMOS logic or Current Mode Logic (CML), is considered. In the first case, the main component of the power consumption is $\mathrm{P}_{\mathrm{AC}}$ and to improve power efficiency it is necessary to implement leakage reduction techniques to decrease $\mathrm{P}_{\mathrm{DC}}$ [18], whereas in the second case the main part comes from $\mathrm{P}_{\mathrm{DC}}$. However, in this case, it is easy to change the power sharing between $\mathrm{P}_{\mathrm{DC}}$ and $\mathrm{P}_{\mathrm{AC}}$ by switching on/off CML gates by

\begin{tabular}{|c|c|c|c|c|}
\hline Application & Location & $\begin{array}{l}\text { Sample } \\
\text { Size } \\
\text { (bit) }\end{array}$ & $\begin{array}{c}\text { Sampling } \\
\text { Frequency } \\
\text { (Hz) }\end{array}$ & $\begin{array}{c}\text { Bit } \\
\text { Rate } \\
\text { (bps) }\end{array}$ \\
\hline $\begin{array}{c}\text { Oxygen Saturation, } \\
\text { Blood Pressure, } \\
\text { Body Temperature }\end{array}$ & On-body & \multirow[t]{2}{*}{16} & \multirow[t]{2}{*}{$1 / 3600$} & \multirow[t]{2}{*}{$4.4 \mathrm{~m}$} \\
\hline $\begin{array}{c}\text { Drug Dosage, } \\
\text { Glucose Monitoring }\end{array}$ & In-body & & & \\
\hline Heart Beat Rate & On-body & 8 & 1 & 8 \\
\hline $\begin{array}{c}\text { ElectroCardioGraphy } \\
\text { (ECG) }\end{array}$ & On-body & 12 & 250 & $3 \mathrm{k}$ \\
\hline $\begin{array}{l}\text { ElectroMyoGraphy } \\
\text { (EMG) }\end{array}$ & On-body & 16 & $4 \mathrm{k}$ & $64 k$ \\
\hline Hearing Aid & On-body & 16 & $22.05 \mathrm{k}$ & $200 \mathrm{k}$ \\
\hline $\begin{array}{l}\text { Deep Brain } \\
\text { Stimulation }\end{array}$ & In-body & - & - & $1 \mathrm{M}$ \\
\hline Capsule Endoscopy ${ }^{\mathrm{b}}$ & In-body & $307.2 \mathrm{k}$ & 6 & $1.8 \mathrm{M}$ \\
\hline $\begin{array}{l}\text { Brain Machine } \\
\text { Interface }\end{array}$ & In-body & - & - & $>10 \mathrm{M}$ \\
\hline
\end{tabular}
disabling their current sources [19] with the help of the baseband manager since pulse generation times are known.

TABLE I. TECHNICAL REQUIREMENTS FOR BIOMEDICAL APPLICATIONS

${ }^{\text {b. }}$ Resolution of $320 \times 320$.

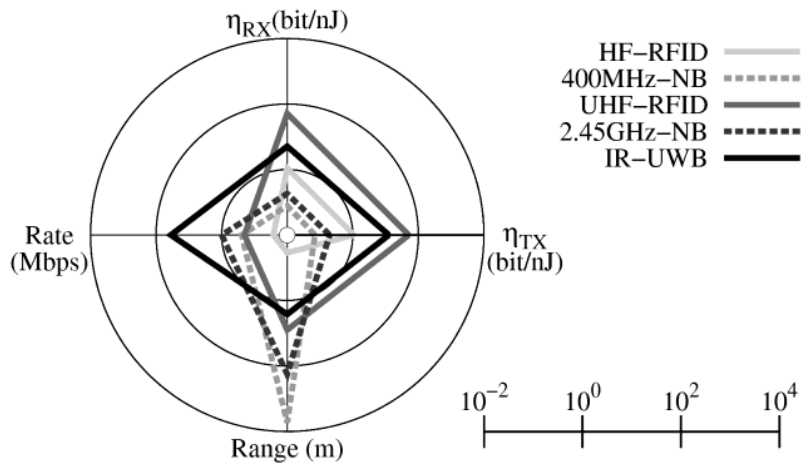

Fig. 1. Wiresless technologies comparison from averaged results of previous published works (HF-RFID: [4][5], 400MHz-NB: [8][9][10], UHF-RFID: [6][7], 2.45GHz-NB: [11][12][13], IR-UWB: [14][15][16]). 

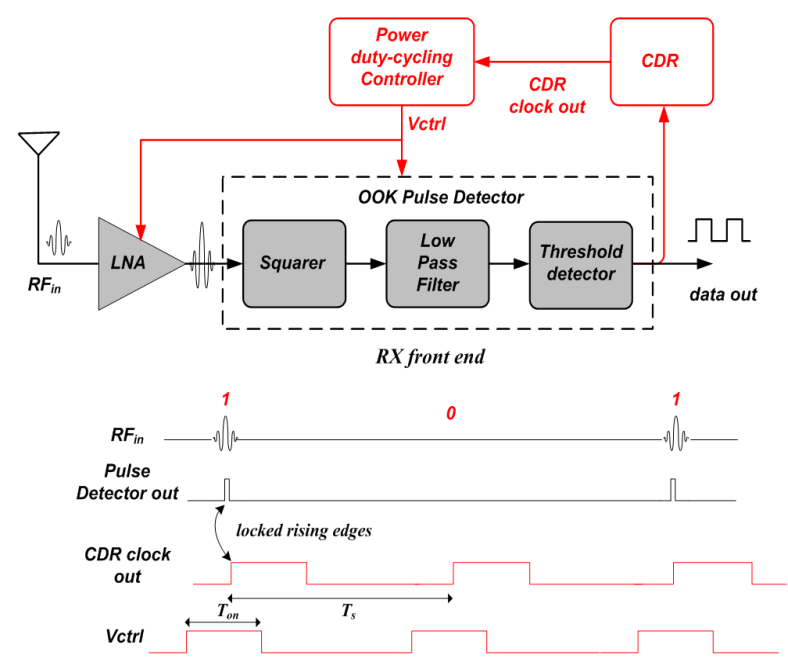

Fig. 2. Self synchronized and duty-cycled receiver architecture.

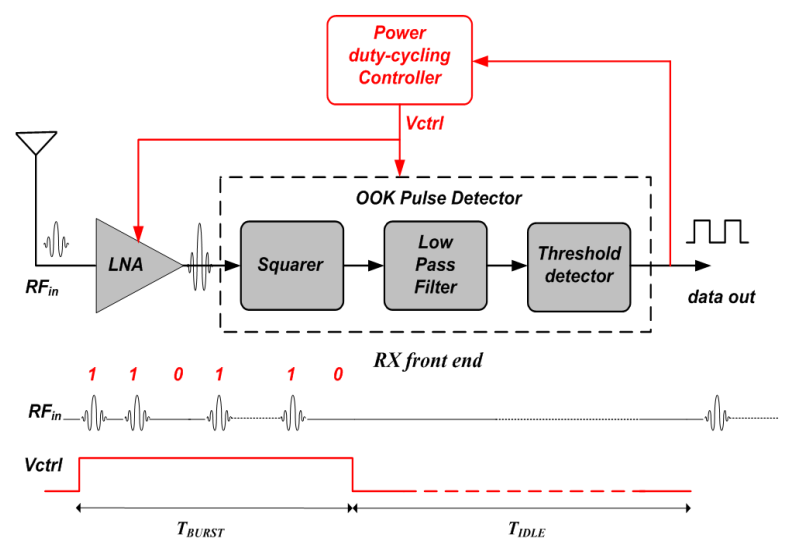

Fig. 3. Burst scale duty-cycled receiver architecture.

\section{Power Management Schemes of IR-UWB receivers}

Low-power consumption can be achieved for both NB and UWB receivers by using very simple architectures. Such architectures operate with OOK modulation scheme in noncoherent receivers when an envelope detector can be used for the demodulation of the encoded data. By using this technic, low power consumption can be achieved for the RF front end at the cost of sensitivity degradation. In [20], a NB receiver is presented with $54 \mu \mathrm{W}$ power consumption at $100 \mathrm{kbps}$ data rate with $-72 \mathrm{dBm}$ sensitivity. An example of usual non-coherent IR-UWB receiver is shown in Fig. 2 and Fig. 3. The circuit is composed of a Low Noise Amplifier (LNA) and an OOK pulse detector. Non-coherent IR-UWB receivers can also achieve low power consumption at low and medium data rate by duty cycling RF front end blocks between two received symbols. [21][22][23] give some examples of duty cycled UWB receivers where microwatt range power consumptions are achieved for low and medium data rates.

Typical architecture of a self-synchronized and duty cycled receiver is given in Fig. 2. A duty cycled operation at the pulse scale which is used here needs the synchronization of the receiver with the emitter. This operation can be achieved by a
Clock and Data Recovery (CDR) which can be implemented with low power consumption [21][22]. However, an acquisition mode time slot, where the RX is always on while receiving a synchronization frame, is necessary. This acquisition mode, which has duration greater than several tens of bits [21], degrades the power consumption efficiency especially when a short amount of data has to be sent. After the acquisition mode cycle, the LNA is duty-cycled with a steady state on time duration $\mathrm{T}_{\text {on. }} \mathrm{T}_{\text {on }}$ has to be set according to the peak to peak CDR jitter, the settling time of the RF front end blocks, and the pulse duration. The settling time of the RF front end blocks is typically around few nanoseconds, while the CDR jitter depends on the symbol rate and is typically equal to tens of nanoseconds at 1Msps. In [21], a self-synchronized and duty cycled UWB receiver exhibits $750 \mu \mathrm{W}$ power consumption for the entire receiver at $2 \mathrm{Mbps}$ data rate with $-76.5 \mathrm{dBm}$ sensitivity.

To avoid the long acquisition mode time slot a duty cycle operation at the frame scale can be used instead of duty cycle operation at the pulse scale. The sequence of regularly spaced pulses is replaced by bursts giving the same mean data rate. To avoid changes in the mean EIRP, PRF MEAN must be kept constant during $1 \mathrm{~ms}$ which is the spectrum analyzer sweep time imposed by Federal Communications Commission (FCC) to measure the mean EIRP. The maximal burst duration is then given by:

$$
T_{B U R S T}[\mathrm{~ms}]=\frac{P R F_{M E A N}}{P R F_{B U R S T}} \times 1 \mathrm{~ms}=\eta_{B U R S T} \times 1 \mathrm{~ms}
$$

and the receiver can be turned off during:

$$
T_{I D L E}[\mathrm{~ms}]=1 \mathrm{~ms}-T_{B U R S T}[\mathrm{~ms}]
$$

By using $\mathrm{PRF}_{\mathrm{BURST}}$ much greater than $\mathrm{PRF}_{\mathrm{MEAN}}$, the frame scale duty cycling ratio $\eta_{\text {BURST }}$ is low and a great improvement in the power consumption can be achieved with relaxed synchronization constraints. The receiver does not also need a CDR and its associated acquisition time slot, since its output (data out) can be sampled and stored in a memory sized according to $\mathrm{T}_{\mathrm{BURST}}$ and $\mathrm{PRF}_{\mathrm{BURST}}$, and demodulated during

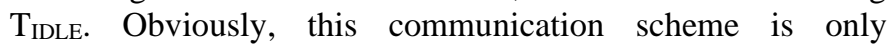
practicable for low and medium mean data rates since the instantaneous data rate is increased. Such frame scale duty cycled receiver architecture is depicted in Fig. 3. The synchronization between the burst position and the turn on command has relaxed constraints and can be achieved by a low power microcontroller where the clock could be synchronized with other clocks of the network using standard synchronized networks protocols or by using an ultra-low power wake up triggering protocol which is out of the scope of this paper.

\section{EXAMPLES OF MEASURED PROTOTYPES}

To illustrate the proposed power saving strategies for IRUWB, two emitters and one receiver are presented. They have been designed using the 130nm CMOS technology from STMicroelectronics and measured under a supply voltage of $1.2 \mathrm{~V}$. 


\section{A. Versatile IR-UWB emitter}

A versatile transmitter with tunable bandwidth has been proposed in [24]. This pulse generator uses elementary pulse combination method which allows an arbitrary pulse to be synthesized according to the targeted bandwidth. As shown in Fig. 4, it is constituted of an elementary pulses generator using a CMOS Voltage Controlled Delay Line (VCDL) and a combination circuit using multiple parallel $\mathrm{H}$-bridges. Its core area is $0.25 \times 0.45 \mathrm{~mm}^{2}$ and its die area is $0.6 \times 1.3 \mathrm{~mm}^{2}$. From measurement results, it is able to generate pulses composed of 1 to 8 elementary pulses with a central frequency between $2.35 \mathrm{GHz}$ and $5.55 \mathrm{GHz}$. When it is programmed to generate a $3-5 \mathrm{GHz}$ pulse, it achieves $0.9 \mathrm{Vpp}$ pulse which leads to an energy emitted by pulse $E_{P}$ of 2.08 pJ on a $100 \Omega$ differential load. In the worst case (when all elementary pulses are set to their maximum values), $\mathrm{P}_{\mathrm{DC}}$ is equal to $0.58 \mathrm{~mW}$ and $\mathrm{E}_{\mathrm{AC}}$ is equal to 209pJ/pulse. As CMOS logic has been only used, $P_{D C}$ is only due to high leakage currents since MOS transistors have been designed large enough to achieve very fast pulses. To stay energy efficient for low data rate applications, leakage reduction methods are required but not implemented here. For high data rate application the maximum energy efficiency is close to $4.78 \mathrm{bit} / \mathrm{nJ}$ whereas it falls to $0.17 \mathrm{bit} / \mathrm{nJ} @ 100 \mathrm{kbps}$.

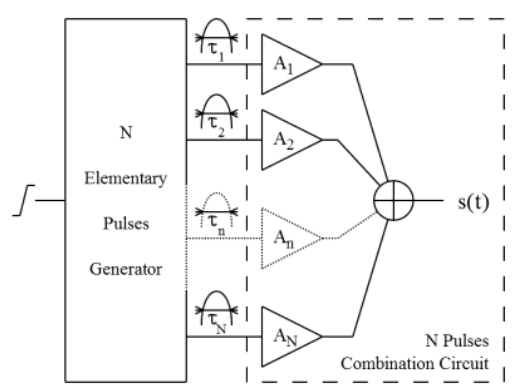

(a)

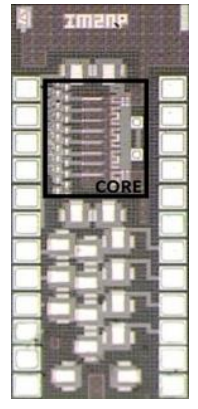

(b)
Fig. 4. Architecture (a) and die photography (b) of the versatile transmitter.

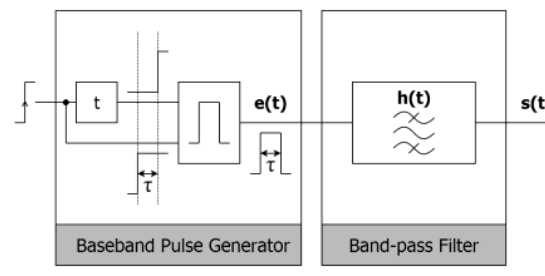

(a)

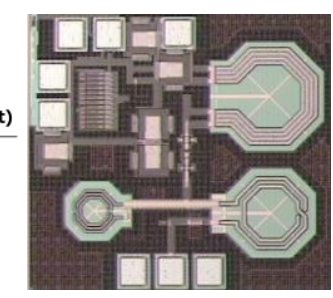

(b)
Fig. 5. Principle (a) and die photo (b) of the ultra low-power transmitter.

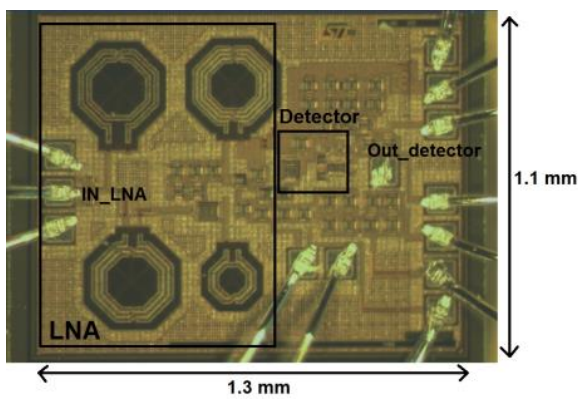

Fig. 6. Die photography of 3-5GHz IR-UWB Front End receiver.

\section{B. Ultra low-power 3.1-10.6GHz emitter}

To reach energy-efficient IR-UWB transmitter for low bit rates and high bit rates, a pulse generator based on filter excitation technique has been presented in [18]. As shown in Fig. 5, this method requires the generation of a baseband pulse which is filtered by a band-pass filter. The baseband pulse generator is constituted of a CMOS VCDL and the band-pass filter is a $3.1-10.6 \mathrm{GHz}$ third order Bessel filter. To reduce the static part of the power consumption and also the leakage currents, an on/off system which uses low leakage MOS transistors has been designed to supply the baseband pulse generator using high speed MOS transistors. The measured pulse generator has a die area of $0.7 \times 0.8 \mathrm{~mm}^{2}$ and exhibits $2 \mathrm{Vpp}$ pulses centered at $6.3 \mathrm{GHz}$ with a $10 \mathrm{~dB}$ bandwidth of $5.2 \mathrm{GHz}$, which leads to an energy emitted by pulse of $2.15 \mathrm{pJ}$ on a $50 \Omega$ load. From measurement, $\mathrm{P}_{\mathrm{DC}}$ is equal to $3.91 \mu \mathrm{W}$ and $\mathrm{E}_{\mathrm{AC}}$ is equal to $68 \mathrm{pJ} /$ pulse which gives a power consumption of $7.3 \mu \mathrm{W}$ at $100 \mathrm{kbps}$ and $3.4 \mathrm{~mW}$ at $100 \mathrm{Mbps}$ for an OOK modulation. This leads to a maximum energy efficiency of $14.7 \mathrm{bit} / \mathrm{nJ}$ when high data rate applications are targeted and an energy efficiency of 13.7bit/nJ@100kbps.

\section{3-5GHz IR-UWB Front End receiver}

In [14], a low power 3-5GHz IR-UWB receiver front-end for WBAN and biomedical applications is proposed. This noncoherent receiver is designed for OOK signaling and uses very simple front end architecture, such as depicted in Fig. 2 and Fig. 3, based on a high gain LC filter LNA followed by a pulse detector composed of a self-mixing squarer, a low pas filter and a threshold detector. This asynchronous front end receiver does not need any external frequency reference. The LC filter LNA provides a second order band pass response helpful for unwanted signals rejection. Since self-mixing squarer conversion gain drops drastically at low input levels, the LNA must provide enough gain to allow the signal to be higher than the noise floor of the following stages at the targeted maximum communication range. For this purpose the voltage gain of the LNA has been maximized resulting in a high power consumption which represents the main contribution of the whole front end receiver consumption. Fig. 6 shows the microphotograph of the die. A summary of the front end receiver performance is given in TABLE II. .

TABLE II. SUMMARY OF 3-5GHz UWB-IR RECEIVER PERFORMANCE

\begin{tabular}{|c|c|}
\hline Parameters & Values \\
\hline Die Area & $1.43 \mathrm{~mm}^{2}$ \\
\hline Bandwidth & $3-5 \mathrm{GHz}$ \\
\hline LNA : Voltage gain \| Noise figure & $35.3 \mathrm{~dB}+/-0.2 \mathrm{~dB} \| 3.8-5 \mathrm{~dB}$ \\
\hline $\begin{array}{l}\text { RX power consumption: } \\
\text { Always On } \| \text { Always off }\end{array}$ & $14.4 \mathrm{~mW} \| 36 \mu \mathrm{W}$ \\
\hline $\begin{array}{l}\text { RX energy consumption } \\
\text { @ maximum data rate }\end{array}$ & 144pJ@100Mbps \\
\hline \multirow{2}{*}{ RX energy efficiency } & 6.94bit/nJ@100Mbps \\
\hline & 0.007bit/nJ@100kbps \\
\hline \multirow{2}{*}{ RX sensitivity@BER=10 $0^{-3}$} & $-59.3 \mathrm{dBm} @ 100 \mathrm{Mbps}$ \\
\hline & -89.3dBm@100kbps \\
\hline $\begin{array}{l}\text { RX minimum consumption in burst } \\
\text { mode for } \mathrm{T}_{\mathrm{BURST}}=1 \mathrm{us}, \mathrm{T}_{\mathrm{FRAME}}=1 \mathrm{~ms}\end{array}$ & 50.4 $\mathrm{WW} 100 \mathrm{kbps}$ \\
\hline $\begin{array}{l}\text { RX energy efficiency in burst mode } \\
\text { for } T_{\text {BURST }}=1 \mathrm{us}, T_{\text {FRAME }}=1 \mathrm{~ms}\end{array}$ & 1.98bit/nJ@100kbps \\
\hline
\end{tabular}


The receiver sensitivity is $-89.3 \mathrm{dBm} @ 100 \mathrm{kbps}$. The receiver power consumption is $14.4 \mathrm{~mW}$ at the maximum data rate of $100 \mathrm{Mbps}$ and shows an energy efficiency of 6.94bit/nJ@100Mbps which falls to 0.007bit/nJ@100kbps. This front end has not been designed to allow pulse scale duty cycling which requires nanosecond scale $\mathrm{T}_{\text {on }}$ time and it is also not possible to optimize the energy efficiency easily. However, it can be easily used with a burst frame duty cycling such as described in Fig. 3. In this case, the receiver could consume only $50.4 \mu \mathrm{W}$ at $100 \mathrm{kbps}$ mean data rate which leads to an energy efficiency of $1.98 \mathrm{bit} / \mathrm{nJ}$ if turn on/off times are considered instantaneous with $\mathrm{T}_{\mathrm{BURST}}$ equals to $1 \mathrm{us}, \mathrm{PRF}_{\mathrm{BURST}}$ equals to $100 \mathrm{Mbps}$, and $\mathrm{T}_{\text {IDLE }}$ equals to $999 \mathrm{us}$. The total frame period is also set to $1 \mathrm{~ms}$ to comply with the FCC requirements for EIRP measurement.

\section{CONLUSION}

Due to the gated nature of IR-UWB communications, it appears that the higher the data rate is, the more energyefficient IR-UWB emitters and receiver are. IR-UWB is intrinsically well-suited for short range healthcare applications when high data rate are required. However, many healthcare applications require low data rate applications. It is then feasible to drastically reduce the static part of the power consumption to achieve an energy efficiency closed to its maximum. To do so, some design strategies for emitters and power management schemes for receivers have been proposed and their efficiency is demonstrated on existing circuits.

\section{REFERENCES}

[1] I. Widya, A. van Halteren, V. Jones, R. Bults, and P. Viergout, "Telematic Requirements for a Mobile and Wireless Healthcare System derived from Enterprise Models," International Conference on Telecommunications, ConTEL, pp. 527-534, 2003.

[2] H. Lee, H. Cho and H.J. Yoo, "A 33uW/node Duty Cycle Controlled HBC Transceiver System for Medical BAN with 64 Sensor Nodes," IEEE Custom Integrated Circuits, CICC, pp. 1-8, 2014.

[3] Suzuki, T. ; Ando, H. ; Yoshida, T.; Sawahata, H.; Kawasaki, K. ;Hasegawa, I. ; Matsushita, K. ; Hirata, M. ; Yoshimine, T. ;Takizawa, K., "Super multi-channel recording systems with UWB wireless transmitter for BMI", Annual International Conference of the IEEE Engineering in Medicine and Biology Society, EMBC, 2014.

[4] Z. Xiao, X. Tan, X. Chen, S. Chen, Z. Zhang, H. Zhang, J. Wang, Y. Huang, P. Zhang, L. Zheng and H. Min, "An Implantable RFID Sensor Tag toward Continuous Glucose Monitoring," IEEE Journal of Biomedical and Health Informatics, vol. 19, no. 3, pp. 910-919, May 2015.

[5] M.M. Ahmadi and G.A. Jullien, "A Wireless-Implantable Microsystem for Continuous Blood Glucose Monitoring," IEEE Transactions on Biomedical Circuits and Systems, vol. 3, no. 3, pp. 169-180, June 2009.

[6] M.H. Nazari, M. Mujeeb-U-Rahman and A. Scherer, "An implantable continuous glucose monitoring microsystem in $0.18 \mu \mathrm{m}$ CMOS," Symposium on VLSI Circuits, pp. 1-2, 2014.

[7] S. Radiom, M. Baghaej-Nejad, G. Vandenbosch, L.R. Zheng and G. Gielen, "Far-field RF powering system for RFID and implantable devices with monolithically integrated on-chip antenna," IEEE Radio Frequency Integrated Circuits Symposium, RFIC, pp. 113-116, 2010.

[8] M.R. Nezhad-Ahmadi, G. Weale, A. El-Agha, D. Griesdorf, G. Tumbush, A. Hollinger, M. Matthey, H. Meiners and S. Asgaran, "A $2 \mathrm{~mW} 400 \mathrm{MHz}$ RF transceiver SoC in 0.18um CMOS technology for wireless medical applications," IEEE Radio Frequency Integrated Circuits Symposium, RFIC, pp. 285-288, 2008.

[9] F. Carrara, A. Italia, G. Palmisano and R. Guerra, "A 400-MHz CMOS radio front-end for ultra low-power medical implantable applications," European Solid-State Circuits Conference, ESSCIRC, pp. 232-235, 2009.

[10] N. Cho, J. Bae and H.J. Yoo, "A $10.8 \mathrm{~mW}$ Body Channel Communication/MICS Dual-Band Transceiver for a Unified Body Sensor Network Controller," IEEE Journal of Solid-State Circuits, vol. 44, no. 12, pp. 3459-3468, December 2009.

[11] M. Vidojkovic, X. Huang, P. Harpe, S. Rampu, C. Zhou, L. Huang, J. van de Molengraft, K. Imamura, B. Busze, F. Bouwens, M. Konijnenburg, J. Santana, A. Breeschoten, J. Huisken, K. Philips, G. Dolmans and H. de Groot, "A $2.4 \mathrm{GHz}$ ULP OOK Single-Chip Transceiver for Healthcare Applications," IEEE Transactions on Biomedical Circuits and Systems, vol. 5, no. 6, pp. 523-534, December 2011.

[12] G. Devita, A.C.W Wong, M. Dawkins, K. Glaros, U. Kiani, F. Lauria, V. Madaka, O. Omeni, J. Schiff, A. Vasudevan, L. Whitaker, S. Yu and A. Burdett, "A 5mW multi-standard Bluetooth LE/IEEE 802.15.6 SoC for WBAN applications," European Solid-State Circuits Conference, ESSCIRC, pp. 283-286, 2014.

[13] Nordic Semiconductor, "nRF51822 SoC for Bluetooth low energy and 2.4GHz ultra low-power wireless applications", Dec. 2013 [Online]. Available: www.nordicsemi.com

[14] O. Ramos Sparrow, R. Vauche, N. Dehaese, S. Bourdel, J. Gaubert, I. Ben Amor, E. Muhr, P. Losco and O. Fourquin, "High rate UWB CMOS transceiver chipset for WBAN and biomedical applications," Analog Integrated Circuits and Signal Processing, vol. 81, no. 1, pp. 215-227, July 2014.

[15] R.K. Dokania, X.Y. Wang, S.G. Tallur and A.B. Apsel, "A Low Power Impulse Radio Design for Body-Area-Networks," IEEE Transactions on Circuits and Systems I, vol. 58, no. 7, pp. 1458-1469, July 2011.

[16] S. Soldà, M. Caruso, A. Bevilacqua, A. Gerosa, D. Vogrig and A. Neviani, "A $5 \mathrm{Mb} / \mathrm{s}$ UWB-IR Transceiver Front-End for Wireless Sensor Networks in $0.13 \mu \mathrm{m}$ CMOS, " IEEE Journal of Solid-State Circuits, vol. 46, no. 7, pp. 1636-1647, July 2011.

[17] D. Morche, G. Masson, S. de Rivaz, F. Dehmas, S. Paquelet, A. Bisiaux, O. Fourquin, J. Gaubert and S. Bourdel, "Double-Quadrature UWB Receiver for Wide-Range Localization Applications With Sub-cm Ranging Precision," IEEE Journal of Solid-State Circuits, vol. 48, no. 10, pp. 2351-2362, October 2013.

[18] R. Vauche, S. Bourdel, N. Dehaese, J. Gaubert, O. Ramos Sparrow, E. Muhr and H. Barthelemy, "High efficiency UWB pulse generator for ultra-low-power applications," International Journal of Microwave and Wireless Technologies, March 2015 [Online]. Available: CJO2015.

[19] R. Vauche, E. Muhr, N. Tall, A. Haloua S. Bourdel, J. Gaubert, N. Dehaese, H. Barthelemy, "Ultra-WideBand Voltage Controlled Oscillator with Commutable Phases for BPSK Implementation," IEEE New Circuits and Systems Conference, NEWCAS, in press, 2015.

[20] N. M. Pletcher, S. Gambini, and J. Rabaey, "A 52uW wake-up receiver with $-72 \mathrm{dBm}$ sensitivity using an uncertain-IF architecture," IEEE J. Solid-State Circuits, vol. 44, no. 1, pp. 269-280, Jan. 2009.

[21] F. Lee and A. Chandrakasan, "A $2.5 \mathrm{~nJ} / \mathrm{bit}$ 0.65V Pulsed UWB Receiver in $90 \mathrm{~nm}$ CMOS," IEEE Journal Of Solid-State Circuits, vol. 42, no.12, pp.2851-2859, December 2007.

[22] R. Dokania, X. Wang, S. Tallur, C.Dorta-Quinones and A. Apsel, "An Ultralow-Power Dual-Band UWB Impulse Radio," IEEE Transactions On Circuits and Systems II: Express Briefs, vol. 57, no. 7, pp. 541545,June 2010.

[23] B. Vigraham and P. Kinget, "A Self-Duty-Cycled and Synchronized UWB Pulse-Radio Receiver SoC With Automatic Threshold-Recovery Based Demodulation", IEEE Journal Of Solid-State Circuits, vol. 49, no. 3, pp.581-594, March 2014.

[24] R. Vauche, S. Bourdel, N. Dehaese, O. Fourquin, and J. Gaubert, "Fully tunable UWB pulse generator with zero DC power consumption," IEEE International Conference on Ultra-Wideband, ICUWB, pp. 418-422, 2009. 